\title{
Measurement of a density profile of a hot-electron plasma in RT-1 with three-chord interferometry
}

\author{
H. Saitoh*, Y. Yano, Z. Yoshida, M. Nishiura, J. Morikawa, Y. Kawazura, T. Nogami, and M. Yamasaki \\ Graduate School of Frontier Sciences, The University of Tokyo, \\ 5-1-5 Kashiwanoha, Kashiwa, Chiba 277-8561, Japan
}

(Dated: April 1, 2015)

\begin{abstract}
The electron density profile of a plasma in a magnetospheric dipole field configuration was measured with a multi-chord interferometry including a relativistic correction. In order to improve the accuracy of density reconstruction, a $75 \mathrm{GHz}$ interferometer was installed at a vertical chord of the Ring Trap 1 (RT-1) device in addition to previously installed ones at tangential and another vertical chords. The density profile was calculated with three-chord interferometry including the relativistic effects for a plasma consisting of hot and cold electrons generated by electron cyclotron resonance heating $(\mathrm{ECH})$. The results clearly showed the effects of density peaking and magnetic mirror trapping in a strongly inhomogeneous dipole magnetic field.
\end{abstract}

PACS numbers: 52.70.-m, 52.55.-s, 94.30.cq

The Ring Trap 1 (RT-1) is a magnetospheric configuration generated by a levitated dipole field magnet $[1,2]$. In a dipole magnetic field, a plasma spontaneously generates a strongly peaked structure $[3,4]$, which can be stable against pressuredriven MHD modes even in bad curvature regions [5]. This is because the plasma is stabilized due to the effects of strong compressibility of dipole field lines and the effects of a magnetic separatrix [6]. Here density and pressure profiles are important parameters in relation to the self-organization of charged particles in the magnetospheric configuration.

As one of initial diagnostic tools, a $75 \mathrm{GHz}$ interferometer has been used at tangential ports of RT- 1 for the measurement of line integrated electron density. In order to investigate the spatial structures of a plasma, we installed another interferometer in 2008, which showed the formation of a strongly peaked density profile [2]. The observation of peaked density profile was also reported in Levitated Dipole eXperiment (LDX) $[4,7]$. In the analysis with the two-chord interferometry in RT- 1 , we assumed that electron density $n_{\mathrm{e}}$ is constant on a magnetic surface. However, $n_{\mathrm{e}}$ can be inhomogeneous on a magnetic surface due to mirror trap effects in the strongly inhomogeneous dipole field. This is especially a case for ECH plasmas because electrons have large perpendicular kinetic energies. Also, in the interpretation of data from microwave diagnostics for hot electron plasmas, a relativistic correction $[8,9]$ is needed. For the accurate measurement of electron density profiles, we installed a new interferometer in RT- 1 . We measured the density profile of a plasma by taking into the effects of density peaking and mirror trapping with a relativistic correction for the hot electron component [10]. In this paper, we focus on the measurement technique and explain the experimental setup of the three-chord interferometry and the data interpretation method.

Figure 1 shows the schematics of RT-1 including the interferometry chords. A superconducting dipole field magnet is located inside the vacuum chamber, which is magnetically

*Present address: Max Planck Institute for Plasma Physics, Boltzmannstraße 2, D-85748 Garching, Germany
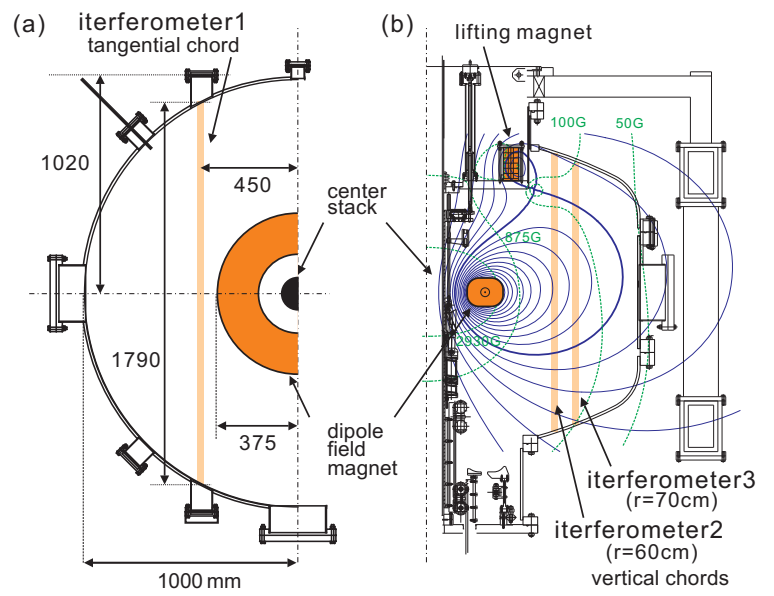

FIG. 1: (color online) (a) Top view and (b) projection view onto the poloidal cross section of RT-1. Interferometers are located at tangential (chord 1) and vertical (chord 2 and 3) ports. Thin lines show magnetic surfaces generated by the combination of levitating and lifting magnets.

levitated by using another lifting magnet located at the top of the chamber [11]. Electron line integrated densities are measured with three sets of heterodyne interferometers. The frequencies of the three interferometers were chosen to be 75.3, 75.4 , and $75.5 \mathrm{GHz}$, so that there are no signaling cross-talks between different chords. As shown in the figure, the interferometer chords are located at vertical (chord 2 and 3 at $r=60$ and $70 \mathrm{~cm}$ ) ports as well as at a tangential (chord 1 at $y=45 \mathrm{~cm}$ ) port. This configuration makes it possible to reconstruct density profiles including the inhomogeneity on magnetic surfaces as well as the peaking effects across magnetic surfaces. Figure 2 shows the typical waveforms of the interferometers. A $8.2 \mathrm{GHz}$ microwave was injected from $t=0$ to $1 \mathrm{~s}$. After the end of microwave injection, plasma density shows a double-exponential type decay because electrons have hot $(\sim 50 \mathrm{keV})$ and cold populations, which can be used for the measurement of the ratio of hot electron component ratio [2].

When a microwave with a frequency $f=2 \pi \omega$ passes through a plasma whose refraction index is $N$, the variation 


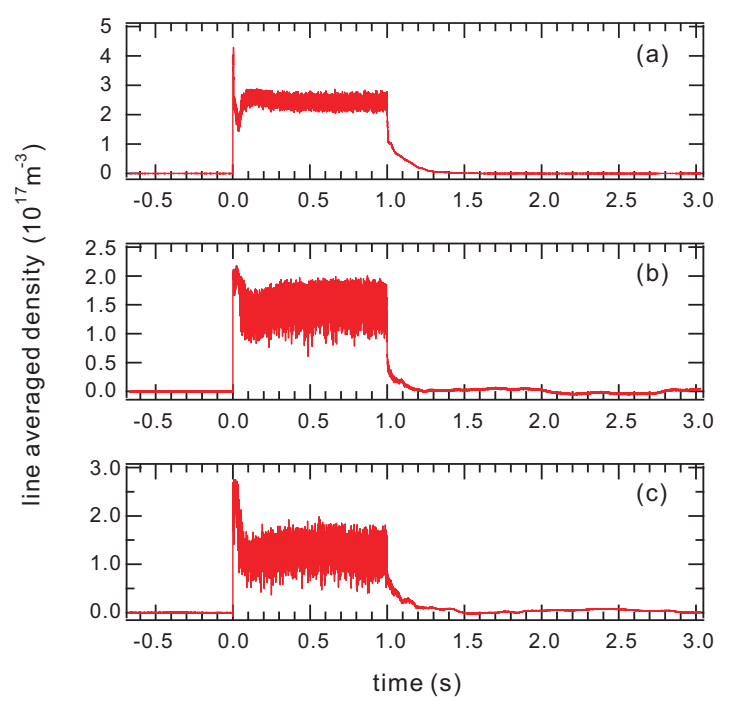

FIG. 2: (color online) Typical waveforms of interferometers located at (a) tangential chord at $y=45 \mathrm{~cm}$, (b) vertical chord at $r=60 \mathrm{~cm}$, and (c) vertical chord at $r=70 \mathrm{~cm}$.

of a phase is

$$
\phi=2 \pi / \lambda \int N d l
$$

Here $\lambda=c / f$ is the wave length of a microwave and $c$ is the speed of light [12]. Because we have interferometers on the vertical chords of the device in addition to one on a tangential chord (Fig. 1), we should consider several modes of microwave propagation in addition to the $\mathrm{O}$-mode. The permittivity tensor of a weakly relativistic plasma is approximated as $[8,9]$

$$
\varepsilon=\left(\begin{array}{ccc}
1-\alpha X /\left(1-Y^{2}\right) & i \beta X Y /\left(1-Y^{2}\right) & 0 \\
-i \beta X Y /\left(1-Y^{2}\right) & -1+\alpha X /\left(1-Y^{2}\right) & 0 \\
0 & 0 & 1-\gamma X
\end{array}\right),
$$

where

$$
\begin{gathered}
\alpha=1-\frac{5}{2 \mu} \frac{1+X}{1-X}, \\
\beta=1-\frac{5}{\mu} \frac{1}{1-X}, \\
\gamma=1-\frac{5}{2 \mu}
\end{gathered}
$$

by using $X=\omega_{\mathrm{p}}^{2} / \omega^{2}$ and $Y=\omega_{\mathrm{ce}} / \omega$, where $\omega_{p}=$ $\left(n_{\mathrm{e}} e^{2} / \varepsilon_{0} m_{\mathrm{e}}\right)^{1 / 2}$ is the plasma frequency and $\omega_{\mathrm{ce}}=e B / m_{\mathrm{e}}$ is the electron cyclotron frequency. Relativistic effects are included in

$$
\mu=m_{0} c^{2} / k_{\mathrm{B}} T_{\mathrm{e}}
$$

where $m_{0}$ is the rest mass of an electron and $k_{\mathrm{B}} T_{\mathrm{e}}$ is the temperature of electrons. When a wave propagates in a direction

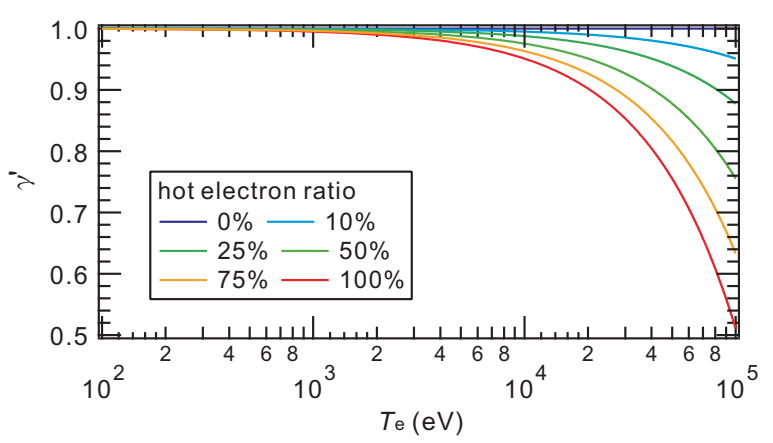

FIG. 3: (color online) A relativistic correction factor, $\gamma^{\prime}$ in (19), in the variation of hot electron temperature $T_{\mathrm{e}}$ for different ratios $r_{\mathrm{hot}}$ of hot electrons.

perpendicular to a magnetic field $\mathbf{B}, N$ of $\mathrm{O}$ and $\mathrm{X}$ modes after relativistic correction are given by

$$
N_{\mathrm{O}}^{2}=1-\gamma X \text { and }
$$

$$
N_{\mathrm{X}}^{2}=\frac{1-2 \alpha X+\alpha^{2} X^{2}-2 Y^{2}+2 \alpha X Y^{2}-\beta^{2} X^{2} Y^{2}+Y^{4}}{\left(-1+Y^{2}\right)\left(-1+\alpha X+Y^{2}\right)} .
$$

For a wave propagating in the parallel direction, $N$ of $R$ and $L$ modes are

$$
\begin{gathered}
N_{\mathrm{R}}^{2}=\frac{-1+\alpha X-\beta X Y+Y^{2}}{-1+Y^{2}} \text { and } \\
N_{\mathrm{L}}^{2}=\frac{-1+\alpha X+\beta X Y+Y^{2}}{-1+Y^{2}} .
\end{gathered}
$$

When the frequency of a diagnostic microwave satisfies

$$
\omega_{\mathrm{p}}^{2}, \omega_{\mathrm{ce}}^{2}<<\omega^{2}
$$

all the reflective indexes in (7)-(10) are approximated as

$$
N=1-\gamma X / 2
$$

independent of the propagation directions. A phase shift observed by an interferometer is given by

$$
\Delta \phi=\frac{2 \pi}{\lambda} \int(1-N) d l=\frac{\pi}{\lambda} \int(\gamma X) d l .
$$

A line density that corresponds to a phase shift of $\Delta \phi=2 \pi$ is then given by

$$
\int n_{\mathrm{e}} d l=7.5 \times 10^{6} \times f / \gamma
$$

Here the units of line density and microwave frequency are $\mathrm{m}^{-2}$ and $\mathrm{s}^{-1}$.

Now we apply this relativistic correction to the interferometry of RT-1. Although microwaves traverse the plasma not entirely in O-mode, as shown in Fig. 1, we may use (12) as the refraction index of the hot electron component of a plasma because the parameters satisfy (11). We denote the ratio of 


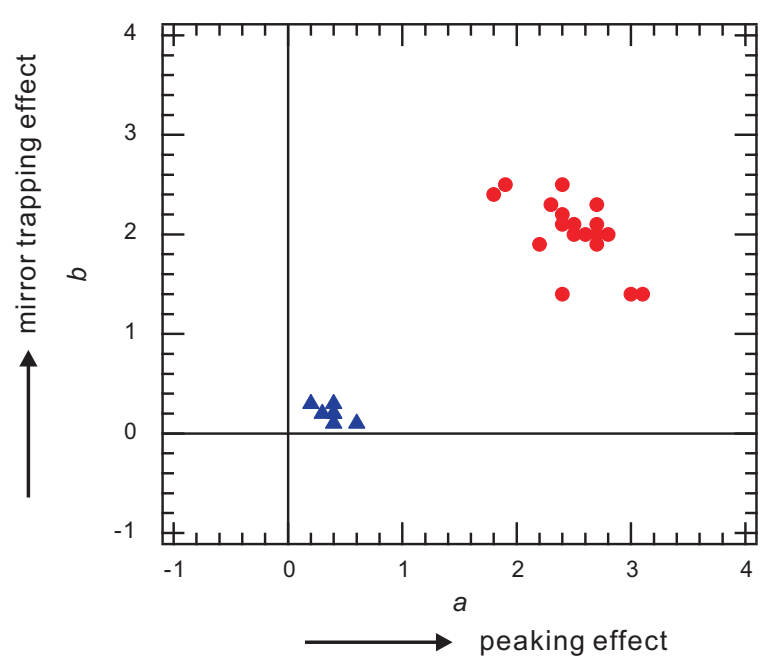

FIG. 4: (color online) The values of $a$ and $b$ when neutral gas pressure $P_{\mathrm{n}}=2.5 \mathrm{mPa}$ (circles), where the ionization degree was close to unity in the main confinement region and when $P_{\mathrm{n}}=15 \mathrm{mPa}$ (triangles), where the neutral effects are significant.

hot and cold electron components in a plasma as $r_{\text {hot }}$ and $r_{\text {cold }}=1-r_{\text {hot }}$. The components of the permittivity tensor is then given by

$$
\begin{gathered}
\varepsilon_{11}=\varepsilon_{22}=1-\left[\alpha r_{\text {hot }}+r_{\text {cold }}\right] \frac{X}{1-Y^{2}}, \\
\varepsilon_{12}=-\varepsilon_{21}=i\left[\beta r_{\text {hot }}+r_{\text {cold }}\right] \frac{X Y}{1-Y^{2}}, \text { and } \\
\varepsilon_{33}=1-i\left[\gamma r_{\text {hot }}+r_{\text {cold }}\right] X .
\end{gathered}
$$

The reflective index for the entire plasma is then

$$
N=1-\left[\gamma r_{\text {hot }}+\left(1-r_{\text {hot }}\right)\right] X / 2 .
$$

When the frequency of the interferometer is $f=75 \mathrm{GHz}$, a line density that corresponds to $\Delta \phi=2 \pi$ is then

$$
\int n_{\mathrm{e}} d l=5.6 \times 10^{17} / \gamma^{\prime}
$$

where $\gamma^{\prime}=\gamma r_{\text {hot }}+r_{\text {cold. }}$. Figure 3 plots the correction factor $\gamma^{\prime}$ in variation of hot electron temperature $T_{\mathrm{e}}$ for various $r_{\text {hot }}$. The relativistic effects become non-negligible when a plasma has a considerable ratio of hot electrons and $T_{\mathrm{e}}$ is above approximately $10 \mathrm{keV}$. In the following analysis, we use the typical hot electron temperature $T_{\mathrm{e}}=50 \mathrm{keV}$ of a high- $\beta$ plasma in RT-1 [2]. The hot component ratio $r_{\text {hot }}$ was estimated by using the decay characteristics of the plasma observed by the interferometry [2].

By using the three line density values, the density profiles were reconstructed with a three parameter model [10] including the effects of density peaking [1] and the mirror trapping effects. It is assumed that the electron density has a dependence on $r$ as

$$
n_{\mathrm{z}=0}(r)=n_{0} r^{-a}
$$
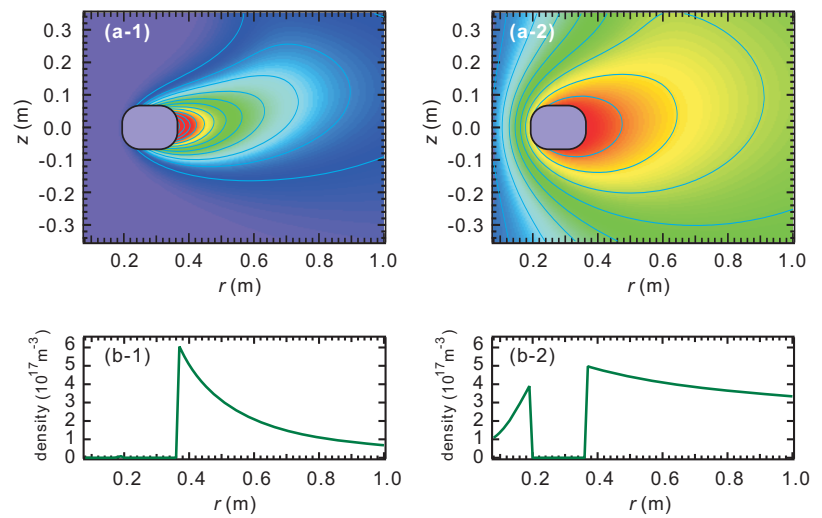

FIG. 5: (color online) Reconstructed electron density profiles (a) on the r-z plane and (b) on the $z=0 \mathrm{~m}$ plane when (1) $P_{\mathrm{n}}=2.5 \mathrm{mPa}$ and (2) $15 \mathrm{mPa}$ by using the averaged values of $a$ and $b$ of circles and triangles in Fig. 4.

on the $z=0 \mathrm{~m}$ plane $\left(r_{\mathrm{c}}<r\right.$, where $r_{\mathrm{c}}$ is the radial position of the dipole field magnet), where $n_{0}$ and $a$ are constants. In order to include the mirror trap effects, we also assume that electron density on a same magnetic surface is given by

$$
n(r, z)=n_{\mathrm{z}=0}(r) \times\left(\frac{B(r, z)}{B_{0}}\right)^{-b}
$$

where $B(r, z)$ is a magnetic field strength and $B_{0}$ is the weakest field strength on the same magnetic surface. We also assume that the plasma has axisymmetric density profiles. By using the values of line densities measured at the three chords, $n_{l 1}$, $n_{l 2}$, and $n_{l 3}$, we find the values of $a$ (density peaking level) and $b$ (strength of mirror trap effects) by the least-square method, minimizing

$$
\Sigma\left(n_{\mathrm{li}}-\int n_{\mathrm{a}, \mathrm{b}}(r, z) d l_{\mathrm{i}}\right)^{2} .
$$

It should be noted that, detailed density profiles between the center stack and the dipole field magnet are still unclear in the present measurements, because all the interferometer chords are located at regions where $r_{\mathrm{c}}<r$.

Figure 4 shows the initial results of the three chord interferometry described above. The values of $a$ and $b$ in (20) and (21) are plotted for low (circles) and high (triangles) filled neutral gas pressure $P_{\mathrm{n}}$. The corresponding density profiles are shown in Fig. 5. When a plasma is almost fully ionized at low $P_{\mathrm{n}}$, both $a$ and $b$ have positive values. It shows that the plasma has a peaked density profile under the influence of the mirror trap in a strongly homogeneous dipole field. When the effects of neutral particles are non-negligible, at higher $P_{\mathrm{n}}, a$ and $b$ are close to zero, indicating that the plasma has a rather flat density distribution. Detailed density profiles between the center stack and the dipole field magnet are still beyond the scope of the present simplified model.

To summarize, we renewed the interferometry system of RT-1 and improved the measurement accuracy of the electron density profiles. By using the three-chord interferometry, 
the effects of mirror trapping were newly included in a density reconstruction model in addition to the peaking effects. We also applied a relativistic correction on the interferometry for plasmas consists of hot and cold electron components. We found that this effect is non-negligible in RT-1, where the plasma is generated and heated by ECH and it contains considerable ratio of hot electrons. When the relativistic effect is not included, it results in the underestimation of line densi- ties typically in $10 \%$. The initial measurements showed that the high- $\beta$ plasmas have peaked density profiles in a strong field regions and they are also strongly affected by the mirror trapping effects of the dipole field, except when the filled gas pressure is very high and the effects of neutral particles are non-negligible.

This work was supported by JSPS KAKENHI Grant Number 23224014 .
[1] Z. Yoshida, H. Saitoh, Y. Yano, H. Mikami, N. Kasaoka et al., Plasma Phys. Cntrl. Fusion 55, 014018 (2013).

[2] H. Saitoh, Z. Yoshida, J. Morikawa, Y. Yano, T. Mizushima et al., Nucl. Fusion 51, 063034 (2011).

[3] Z. Yoshida, H. Saitoh, J. Morikawa, S. Watanabe, Y. Yano et al., Phys. Rev. Lett. 104, 235004 (2010).

[4] A. C. Boxer, R. Bergmann, J. L. Ellsworth, D. T. Garnier, J. Kesner, M. E. Mauel, and P. Woskov, Nature Phys. 6, 207 (2010).

[5] A. Hasegawa, Comm. Plasma Phys. Contr. Fusion 11, 147 (1987).

[6] M. Furukawa, H. Hayashi, and Z. Yoshida, Phys. Plasmas 17, 22503 (2010).
[7] A. C. Boxer, D. T. Garnier, and M. E. Mauel, Rev. Sci. Instrum. 80, 043502 (2009).

[8] E. Mazzucato, Phys. Fluids B 4, 3460 (1992).

[9] H. Hojo, A. Mase, K. Kawahata, Plasma Fusion Res. 4, 010 (2009).

[10] H. Saitoh, Y. Yano, Z. Yoshida, M. Nishiura, J. Morikawa, Y. Kawazura, T. Nogami, and M. Yamasaki, Phys. Plasmas 21, 082511 (2014).

[11] Y. Ogawa, J. Morikawa, T. Mito, N. Yanagi, M. Iwakuma et al., J. Plasma Fusion Res. 79, 643 (2003).

[12] I. H. Hutchinson, Principles of Plasma Diagnostics, 2nd Edition, Cambridge University Press (2002). 